Journal of Engineering Sciences, Assiut University, Vol. 37, No. 5, pp. 1169-1179, September 2009.

\title{
SCATTERING OF A FOCUSED GAUSSIAN BEAM BY A DIELECTRIC COATED SPHEROIDAL PARTICLE
}

\author{
Elsayed Esam M. Khaled \\ Elec. Eng. Dept., Faculty of Engineering, Assiut University, \\ esamk54_2000@hotmail.com
}

(Received August 2, 2009 Accepted August 26, 2009).

\begin{abstract}
Scattering of an on- or off-axis focused Gaussian beam by a coated spheroidal particle (either a prolate or an oblate) is presented. A technique combines the plane-waves spectrum and the T-matrix methods are used to solve the scattering problem. Results are shown for different beam focusing positions with respect to the particle and for different particle's shapes (sphere, and prolate and oblate spheroids) of large size parameters, and for different core's radius or shell's thickness.

The angular scattering intensities are affected by the changes of the core's radius in the case of spheroidal particle more than that for the case of a spherical particle resonates at a low-order mode. As the beam moves farther away from the center of the spheroid the effect of the core on the scattering intensity decreases. A coated spheroidal particle is much affected by changing the beam focusing position than that in the case of a homogeneous spheroidal particle.
\end{abstract}

\section{INTRODUCTION}

Many types of particles in light scattering processes can be modeled as coated or layered spheroidal particles. For example the shape of plant and animal cells, algal and bacteria, microcavities, aerosols in the atmosphere, acid rain droplets, and encapsulated material can be modeled as multi-layered spheroidal objects. A Layered spheroidal particle is important in modeling and studying the absorption of electromagnetic waves emitted by mobile phones in a human head [1]. In cases of coated spheroidal particles the coat serves different purposes in different applications. In pharmaceuticals the coat may help the enclosed drug reach its desired destination, and it may control the time of release. In photography a coat of one type of silver halide on another type of silver halide may enhance the sensitivity of the particle to certain frequencies of light. In nonlinear optical applications, a metallic core or coat may enhance the optical fields in specific regions of the particle [2]. Moreover the coated spheroidal particle is used to investigate the effect of the spheroidal dielectric covers on a radar and communication antennas [3].

The electromagnetic plane wave scattering by a conducting spheroidal object with a dielectric coating was analyzed by Sebak and Sinha [4] using the separation of variables method in a Spheroidal coordinate system. Li et al [5], solved the problem of the electromagnetic radiation from a prolate spheroidal antenna enclosed in a confocal radome (spheroidal dielectric covers). They represented the solution in terms of prolate spheroidal wave functions through the use of separation of variables of the scalar wave equation in a prolate spheroidal coordinate system. Barton [6] calculated the internal 
and near surface electromagnetic fields for a layered confocal spheroidal particle with an arbitrary illumination. Zhang and Han [7] investigated the scattering of a multilayered spheroid with non-confocal and confocal boundaries illuminated with a shaped beam. Peterson and Strom [8] generalized the T- matrix technique which is based on the extended boundary condition method (EBCM) to solve the scattering problem of the confocal coated spheroids illuminated with a plane wave. Khaled et al [2,9] used the technique of T-matrix method with an algorithm devised by Toon and Ackerman to calculate light scattering by a coated sphere illuminated with a focused and shifted Gaussian beam. Quirantes [10] calculated the T-matrix elements of a coated, axisymmetric, nonspherical particle.

In this paper the scattered and internal field intensities of a coated spheroidal particle illuminated with an on- or off-axis focused Gaussian beam are calculated. The T-matrix method is modified using the LISA algorithm [10] to calculate the elements of the T- matrix of a coated spheroid. We show that the angular scattering intensities depend on the core's radius and the shell's thickness, and on the beam position with respect to the coated particle. The new technique can be used to calculate internal and scattered intensities of a larger size parameter spheroidal particle than those described in the literature. Also the technique can be used for an arbitrarily-shaped incident beam shifted to a larger distance from the particle's surface. To check our technique the calculated results are contrasted with those for a coated sphere (Ref. [9]), homogeneous spheroids (Refs. [3, 11, and 12]). Moreover the elements of the T-matrix are checked with those calculated by LISA algorithm for many cases. No differences were noticed in all the cases. Some of these comparisons are presented in the results section. The method used here and the mathematical analysis are outlined in Section 2. Computed results of the angular scattering and internal intensities for a coated spheroidal particle (prolate or oblate) at different core's radius illuminated with a Gaussian beam focused at different positions are presented in Section 3. Conclusions are given in Section 4.

\section{THEORETICAL ANALYSES}

Figure 1 shows a coated spheroidal particle centered at the origin of a right-handed Cartesian coordinate system $(x, y, z)$. The spherical coordinate system $(r, \theta, \varphi)$ is also shown. The radii of the core and the shell at any angle $\theta$ are $r_{c}$ and $r_{s}$ respectively. Those radii for the core and the shell along the $z$-axis are $a_{c}$ and $a_{s}$ whereas along the $x$ axis are $b_{c}$ and $b_{s}$ respectively. For a prolate spheroidal particle the axial ratio $\rho=a_{s} / b_{s}=a_{c} / b_{c}>1$ whereas for an oblate spheroid $\rho<1$. The ratio of the radius of the core to that of the shell (coat) at any angle $\theta$ is $R=r_{c} / r_{s}$. This ratio is considered constant for a certain particle, that is $R=r_{d} / r_{s}=a_{d} / a_{s}=b_{d} b_{s}$, which means that the thickness of the shell is not constant all over the surface of the spheroid. The shell is thicker in the direction of the spheroid's elongation. The particle is illuminated with a lowest order $\left(T E M_{o o}\right)$ monochromatic Gaussian beam (it could be of different shapes) propagates in the z-direction and polarized in the $x-z$ plane. The wavelength of the beam is $\lambda$ and its minimum spot size is $w_{o}$. The focal point of the incident beam is located at an arbitrary point $\left(x_{o}, y_{o}, z_{o}\right)$. For an on-axis beam $x_{o}=y_{o}=z_{o}=0$. The incident Gaussian beam can be expressed in terms of vector spherical harmonics (VSH) as [9]

$$
\mathbf{E}^{i n c}(k \mathbf{r})=H \sum_{m} \sum_{n} D_{m n}\left[a_{e m n}^{t} \mathbf{M}_{e m n}^{1}(k \mathbf{r})+a_{o m n}^{t} \mathbf{M}_{o m n}^{1}(k \mathbf{r})+b_{e m n}^{t} \mathbf{N}_{e m n}^{1}(k \mathbf{r})+b_{o m n}^{t} \mathbf{N}_{o m n}^{1}(k \mathbf{r})\right\rfloor
$$


where $H$, and $D_{m n}$ are constants depend on the incident beam. The $a_{e m n}^{t}, a_{o m n}^{t}, b_{e m n}^{t}$, and $b_{o m n}^{t}$ are the incident field expansion coefficients, and $M^{l}{ }_{e m n}, M_{o m n}^{l}, N_{e m n}^{I}$, and $N^{I}{ }_{\text {omn }}$ are VSH of the first kind, where $m$ (in italic) is the azimuthal mode index and $n$ is the mode number. The symbols $e, o$ stands for even and odd respectively. The wave number is $k=2 * \pi / \lambda$. In the case of a unit amplitude incident plane wave, $H$ is unity and the azimuthal mode index $m=1$.

All the parameters and details of the analysis are given in Ref. [9]. The internal electric field $E^{\text {int }}$ and the scattered electric field $E^{s}$ of a homogeneous arbitrary axisymmetric dielectric object illuminated with a Gaussian beam using the $T$-matrix method are given, respectively by [11]

$$
\begin{aligned}
& \mathbf{E}^{\text {int }}(\mathrm{m} k r)=H \sum_{m} \sum_{n}\left[c_{e m i}^{t} \mathbf{M}_{e m i}^{1}(\mathrm{mk} \mathbf{r})+c_{o m n}^{t} \mathbf{M}_{o m n}^{1}(\mathrm{mk} \mathbf{r})+d_{e m i n}^{t} \mathbf{N}_{e m n}^{1}(\mathrm{mk} \mathbf{r})+d_{o m n}^{t} \mathbf{N}_{o m n}^{1}(\mathrm{mk} \mathbf{r})\right] \\
& \mathbf{E}^{s}(k \mathbf{r})=H \sum_{m} \sum_{n} D_{m n}\left[f_{\text {emn }}^{t} \mathbf{M}_{\text {emn }}^{3}(k \mathbf{r})+f_{\text {omn }}^{t} \mathbf{M}_{\text {omn }}^{3}(k \mathbf{r})+g_{\text {emn }}^{t} \mathbf{N}_{\text {emn }}^{3}(k \mathbf{r})+g_{\text {omn }}^{t} \mathbf{N}_{\text {omn }}^{3}(k \mathbf{r})\right]
\end{aligned}
$$

All the parameters are given in Ref. [11]. The internal field expansion coefficients $c_{e m n}^{t}, c_{o m n}^{t}, d_{e m n}^{t}$, and $d_{o m n}^{t}$ and the scattered field expansion coefficients $f$ ${ }_{\text {emn }}^{t}, f_{\text {omn }}^{t}, g_{\text {emn }}^{t}$, and $g_{\text {omn }}^{t}$ are related to the incident field expansion coefficients through the $A$ - and the $B$-matrices. The elements and properties of these matrices are given in Ref. [11]. The two matrices are related to each other by $[T]^{t}=-[B][A]^{-1}$. Details of the mathematical analysis can be found in Ref. [11]

For a two-layers (or a coated) spheroidal particle with refractive indices $m_{c}$ (core) and $m_{s}$ (shell), the $T$-matrix of the particle can be calculated as [10]

$$
T=-B * A^{-1}=-\left[B_{s}+B_{c s} *\left(-B_{c}^{*} A_{c}^{-1}\right)\right] *\left[A_{s}+A_{c s} *\left(-B_{c}^{*} A_{c}^{-1}\right)\right]^{-1}
$$

where:

(1) $A_{c}$ and $B_{c}$ matrices are calculated for a homogeneous particle with refractive index $\mathrm{m}=\mathrm{m}_{\mathrm{c}} / \mathrm{m}_{\mathrm{s}}$ and a size parameter $\mathrm{x}=\mathrm{x} * \mathrm{R} * \mathrm{~m}_{\mathrm{s}} ; \mathrm{x}=2 * \pi * a_{s} / \lambda$.

(2) $A_{s}$ and $B_{s}$ matrices, are calculated using the refractive index equals to $\mathrm{m}_{\mathrm{s}}$ for the inner and outer layers i.e. $\mathrm{m}_{\mathrm{c}}=\mathrm{m}_{\mathrm{s}}$ for the coated particle.

(3) matrices $A_{c s}$ and $B_{c s}$ are calculated by the same way as $A_{s}$ and $B_{s}$ except that the Bessel functions of the first kind are replaced by Hankel functions.

The programming codes which have been used to calculate the internal and scattered intensities for a homogeneous spheroidal particle [12] are adapted to calculate; first the $A_{c}$ and $B_{s}$ matrices for the inner layer, then the $A_{s}, B_{s}, A_{c s}$, and $B_{c s}$ matrices for the outer layer. Once the elements of these matrices for a coated spheroidal particle are computed they can be used to calculate either internal or scattered field intensities for any beam illumination.

\section{NUMERICAL RESULTS}

To confirm the computations of the present technique the angular scattering intensities are calculated and compared for the same cases presented in Ref. [2] for a coated spherical particle. A coated sphere (axial ratio $\rho=a / b$ in the present programming codes is set to unity) with $R=a_{d} / a_{s}=0.7$ and refractive indices $\mathrm{m}_{\mathrm{s}}=1.36$ and $\mathrm{m}_{\mathrm{c}}=1.5$ for the shell and the core respectively. The size parameter of the homogeneous sphere with no 
core $\left(\mathrm{m}_{\mathrm{c}}=\mathrm{m}_{\mathrm{s}}=1.36\right)$ is $x=34.6469129$ which corresponding to a resonant mode $\mathrm{TE}_{41,1}$ (is a low-order MDR). The coated sphere is illuminated with a focused Gaussian beam of a spot size $w_{o}=1 \mu m$, and $\lambda=0.532 \mu m$. The radius of the shell is $\mathrm{a}_{s}=x * \lambda / 2 \pi=2.933569$. The angular scattering intensities are calculated as a function of the spherical coordinate angle $\theta$ in the $y-z$ plane at points $(500 a, \theta, 90)$ for different beam focusing positions. The computed results are presented in Fig. 2. Results from Ref. [2] for the same cases are also included in the figure for comparison. No differences are noticed. Moreover results (not shown here) for the cases similar to those presented in Refs. [6], [10] are computed for comparison. No differences are found.

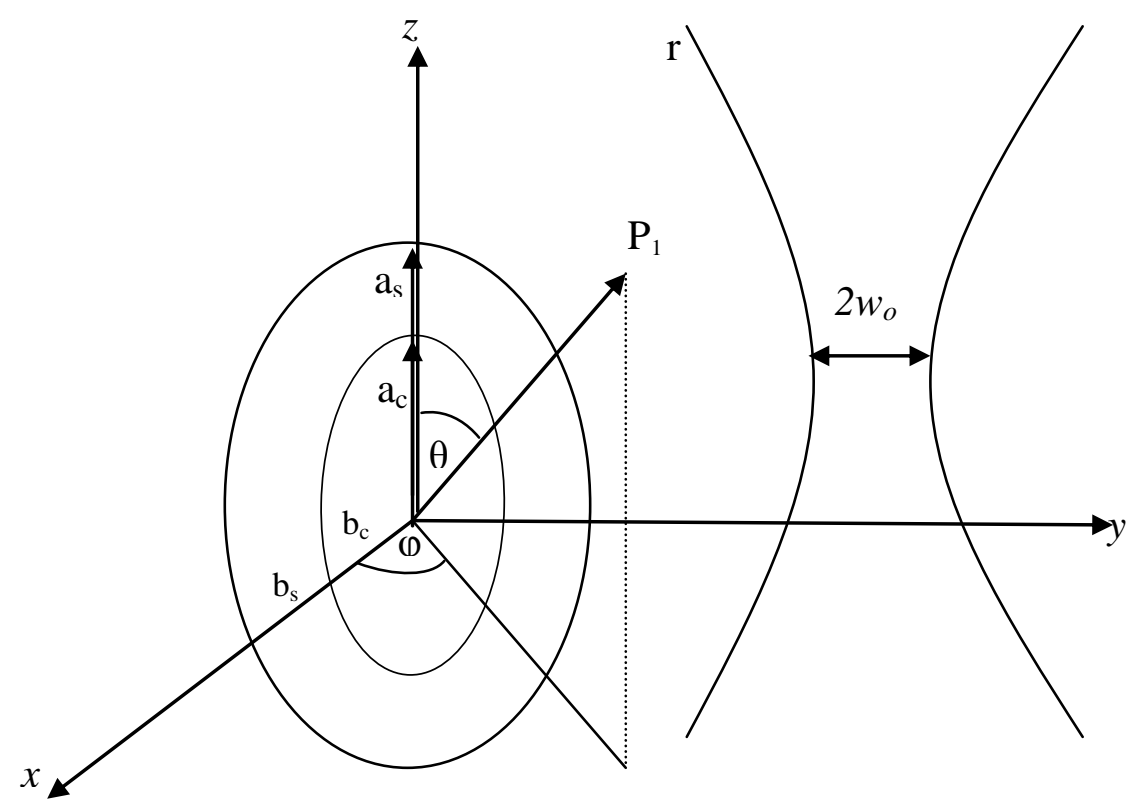

Fig. 1. A coated spheroidal particle centered at the origin of a Cartesian coordinate system $(x, y, z)$ illuminated with a focused shifted Gaussian beam of a minimum spot size $w_{o}$ and propagates in the $z$-direction.

For more illustrations a coated prolate spheroidal particle is considered. The axial ratio of the particle is $\rho=a_{c} / b_{c}=a_{s} / b_{s}=1.4$ with size parameter $x=2 \pi a_{s} / \lambda$ $=47.30942279$ with $\mathrm{a}_{\mathrm{s}}=8.01142 \mu \mathrm{m}$. The refractive indices are $m_{s}=1.36$ and $m_{c}=1.5$. The particle is illuminated with an on-axis Gaussian beam of the same parameters as those in Fig. 2 except that the wavelength is $\lambda=1.064 \mu \mathrm{m}$ and $w_{c}=2 \mu \mathrm{m}$. The angular scattering intensities are computed for different core's radius. The results are also calculated for a homogeneous prolate spheroid of the same radii as those of the shell and with $m_{c}=m_{s}=1.36$, which is similar to that presented in Ref. [12]. The computed results are shown in Fig 3. No differences are noticed between the results of the homogeneous spheroid case and those presented in Ref. [12]. Figure 3 show that the angular scattering intensities contain more number of ripples in the coated spheroid case than those in the case of the homogeneous spheroid. This is due to multireflections which occur in the shell's region. 


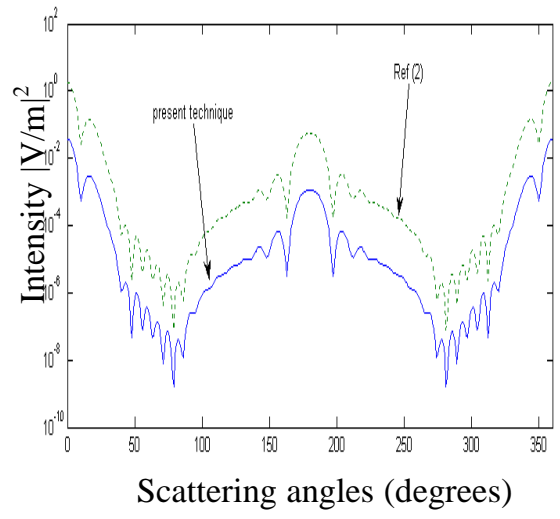

(a)

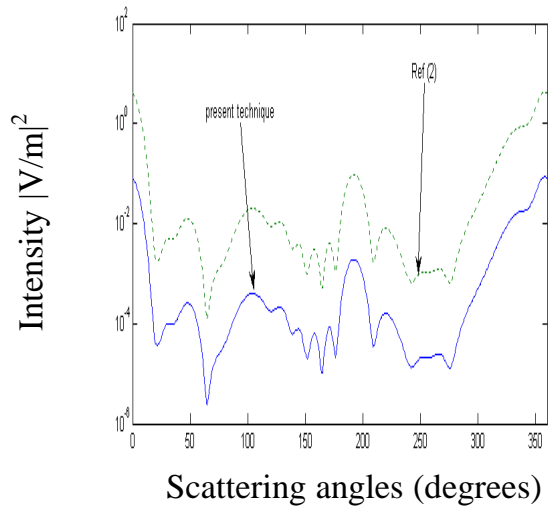

(b)

Fig. 2. Angular scattering intensities as a function of the angle $\theta$ of a coated sphere $(\rho=1.0)$ of $R=a_{c} / a_{s}=0.7$, and refractive indices $\mathrm{m}_{\mathrm{s}}=1.36, \mathrm{~m}_{c}=1.5$ for the shell and the core respectively. The size parameter of the sphere is $x=2 \pi a_{s} / \lambda=34.6469129$. The sphere is illuminated with a Gaussian beam of a minimum spot size $w_{0}=1 \mu \mathrm{m}$, and a wavelength $\lambda=0.532 \mu \mathrm{m}$ propagating in the z-direction. The results are calculated in the $\mathrm{y}-\mathrm{z}$ plane at points $P_{l}(500 a, \theta, 90)$. Results of the same cases in the Ref. [2] are included for comparison, (a) on-axis illumination, (b) the beam is shifted to $\mathrm{x}_{0}=\mathrm{z}_{0}=0$ and

$$
\mathrm{y}_{\mathrm{o}}=0.5 \mathrm{a}_{\mathrm{s}} \text {. }
$$

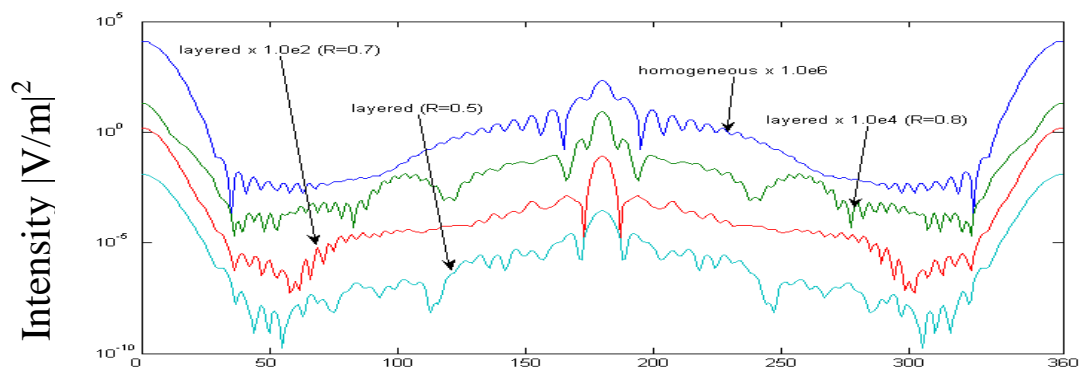

Scattering angles (degrees)

Fig. 3. Angular scattering intensities for a homogeneous and a coated prolate spheroidal particle illuminated with an on-axis Gaussian beam. All the parameters of

the beam are the same as in Fig. 2 except that the wavelength is $\lambda=1.064 \mu \mathrm{m}$ and

$w_{c}=2 \mu \mathrm{m}$. The refractive indices of the shell and the core are $\mathrm{m}_{s}=1.36, \mathrm{~m}_{c}=1.5$ respectively. The axial ratio of the spheroid is $\rho=a_{s} b_{s}=a_{c} b_{c}=1.4$ and $a_{s}=8.011419 \mu \mathrm{m}$. The refractive index of the homogeneous spheroid $\mathrm{m}=\mathrm{m}_{s}=\mathrm{m}_{c}=1.36$ with same radii as those of the shell. Results are shown for different fractions $R=a_{c} / a_{s}=b_{c} / b_{\mathrm{s}}$.

Angular scattering intensities are calculated for the same cases as those considered previously in Fig. 3 except that the incident beam is shifted along the $x$ axis. The results are computed for two beam's shifts $x_{o}=b_{s} / 2$, and $x_{o}=b_{s}$ and illustrated in Fig. 4(a), and 4(b) respectively. Number of ripples in the intensities becomes less pronounced as the beam shifted away from the spheroid's center for both cases 
homogeneous and coated prolate spheroids. As the core's radius increases the number of ripples and the changes in its amplitudes become noticeable. As the core's radius decreases the angular scattering intensity approaches to those of the homogeneous spheroid of the same properties of the shell. Each core's radius case has its own angular scattering distributions. These results can be considered as finger prints for the core's radius which is useful in particle characterization and counting.

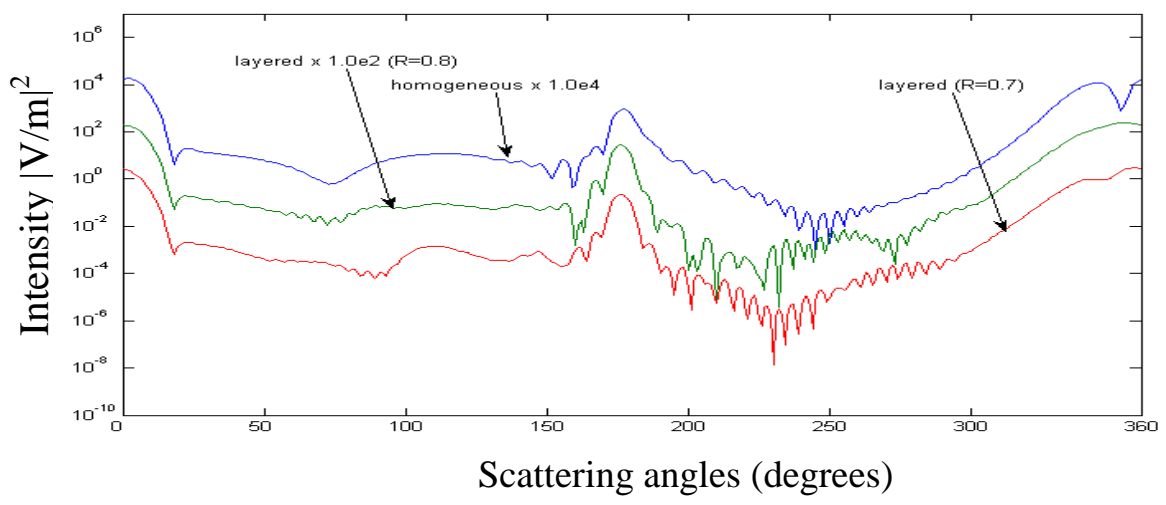

(a)

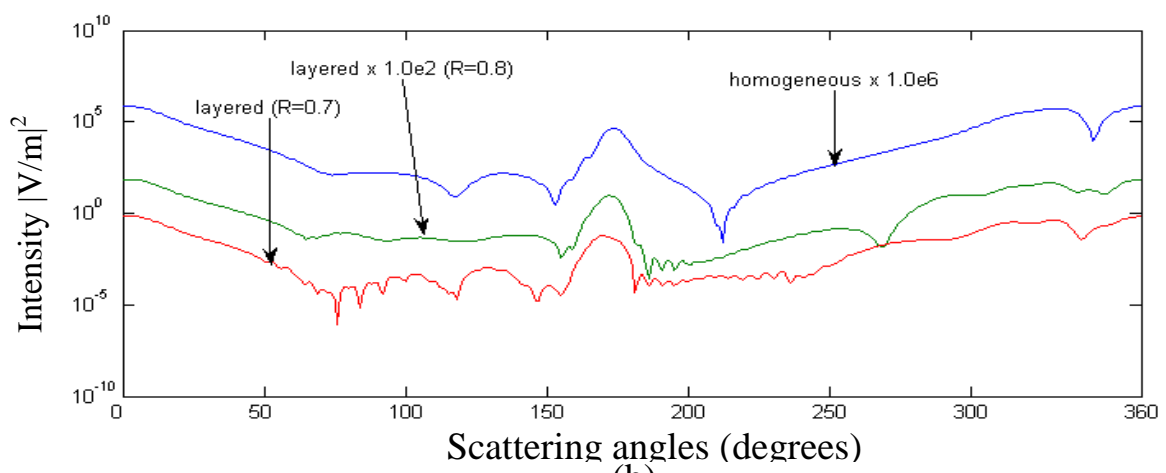

(b)

Fig. 4. Angular scattering intensities for a homogeneous and a coated prolate spheroidal particle illuminated with an off-axis Gaussian beam. All the parameters are the same as in Fig. 3 except that the beam is shifted along the x-axis to (a) $x_{o}=0.5 b_{s}$, and (b) $x_{o}=b_{s}$.

A coated oblate spheroidal particle is considered. The axial ratio of the particle is $\rho=a_{d} / b_{c}=a_{s} / b_{s}=0.7143$ and $x=2 \pi a_{s} / \lambda=33.79244991$ with $a_{\mathrm{s}}=5.72244 \mu \mathrm{m}$. The refractive indices are $m_{s}=1.36, m_{c}=1.5$. The particle is illuminated with a Gaussian beam of the same parameters as those in Fig. 3. The angular scattering intensities are computed for the spheroid for different core's radius. The results are also calculated for a homogeneous oblate spheroid of the same radii as those of the shell and with $m_{c}=m_{s}=1.36$ (similar to the homogeneous spheroid presented in Ref. [12]). The computed results are shown in Figs. 5 and 6 for the on- and off-axis beam illumination respectively. Also no differences are noticed between the results of the homogeneous oblate spheroid and those of the same case presented in Ref. [12]. Figures 5 and 6 show that for the on-axis illumination case the changes in the core's radius have less effects 
on the angular scattering distribution than for the case of prolate spheroidal particle. This smaller effect because the shell's thickness is narrower in the direction of the beam illumination for the oblate spheroidal particle case. The amplitude of changes in the intensities in the backscattering direction $\left(\theta \approx 180^{\circ}\right)$ are less than those in the case of the prolate. These results may be very useful for experimentalists working in the nano-technology industries areas such as particle characterization and counting, semiconductor wafer cleaning, and computer chipsets.

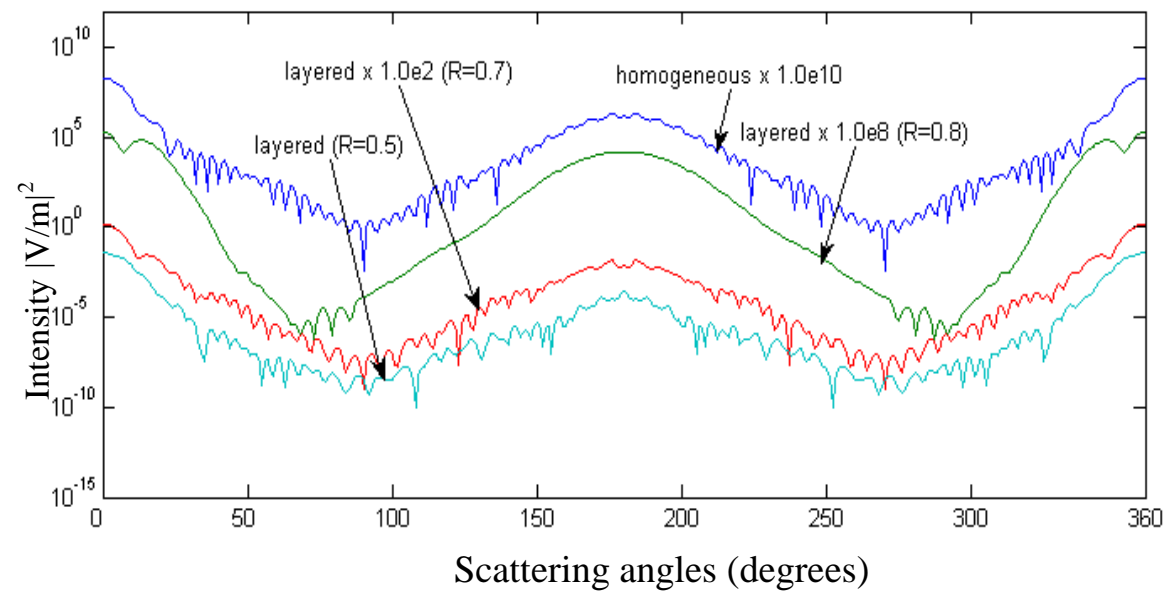

Fig. 5. Angular scattering intensities for a homogeneous and a coated oblate spheroidal particle illuminated with an on-axis Gaussian beam. All the parameters of the beam are the same as in Fig. 3.

The refractive indices of the shell and the core are $\mathrm{m}_{s}=1.36, \mathrm{~m}_{c}=1.5$ respectively. The axial ratio of the prolate spheroid is $\rho=a_{s} / b_{s}=a_{c} / b_{c}=0.7143$ and $a_{s}=5.72244 \mu \mathrm{m}$. The refractive index of the homogeneous spheroid is $\mathrm{m}=\mathrm{m}_{s}=\mathrm{m}_{c}=1.36$. Results are shown for different fractions $R=a_{c} / a_{s}=b_{c} / b_{\mathrm{s}}$.

The effects of the shape of the coated particle and changes of the core's radius on the field intensity are investigated. The summation of the amplitude of the internal field coefficients corresponding to the resonant mode $T E_{58,1}$ over the azimuthal modes that is $\left(\sum_{m}\left|c_{\text {emn }}^{t}\right|+\left|c_{\text {omn }}^{t}\right|\right)$ as a function of the fraction $R=a_{c} / a_{s}=b_{c} / b_{s}$ is computed and illustrated in Fig.7. Different particle's shapes (a coated sphere, coated prolate, and coated oblate) are considered. The refractive indices of the shell and the core are $\mathrm{m}_{\mathrm{c}}=1.5$, and $\mathrm{m}_{\mathrm{s}}=1.36$ respectively for the three particles. The size parameter of a homogeneous sphere having the same properties of the shell with $\mathrm{m}_{\mathrm{c}}=\mathrm{m}_{\mathrm{s}}=1.36$ is $x_{s}=47.3094299$. That size parameter is corresponding to the resonant mode $T E_{58,1}$ MDR. The radius of the shell of the coated sphere can be calculated as $a_{s}=x_{s} *$ $\lambda /(2 * \pi)=8.011419 \mu \mathrm{m}$ for $\lambda=1.064 \mu \mathrm{m}$. The sphere is illuminated with a focused Gaussian beam of the same parameters as those in Fig. 3 except that the beam is shifted to $x_{o}=11.0981 \mu \mathrm{m}>>b_{s}$. Then the axial ratio of the sphere $\left(\rho=a_{s} / b_{s}=a_{c} / b_{c}=1\right)$ is decreased to 0.77799 as an oblate spheroid case and increased to 1.4 an a prolate spheroid case. The results are computed for the three cases. Figure 7 shows that in the case of the sphere the field distribution is corresponding to the resonant mode $T E_{58,1}$ which is a low-order mode. Since the internal field of the low-order resonant mode 
concentrates inside the sphere close to its surface, consequently a small core will not affect the field. Therefore the computed summation is constant up to a certain value of the core's radius at which the core start to interact with the field. As the core's radius increases the sphere becomes off resonance and the amplitude of the coefficients drops to lower values. The effects of changing the core's radius for the prolate spheroid case are more pronounced than for the oblate case since the core is closer to the incident field (small shell's thickness) for the prolate spheroid case.

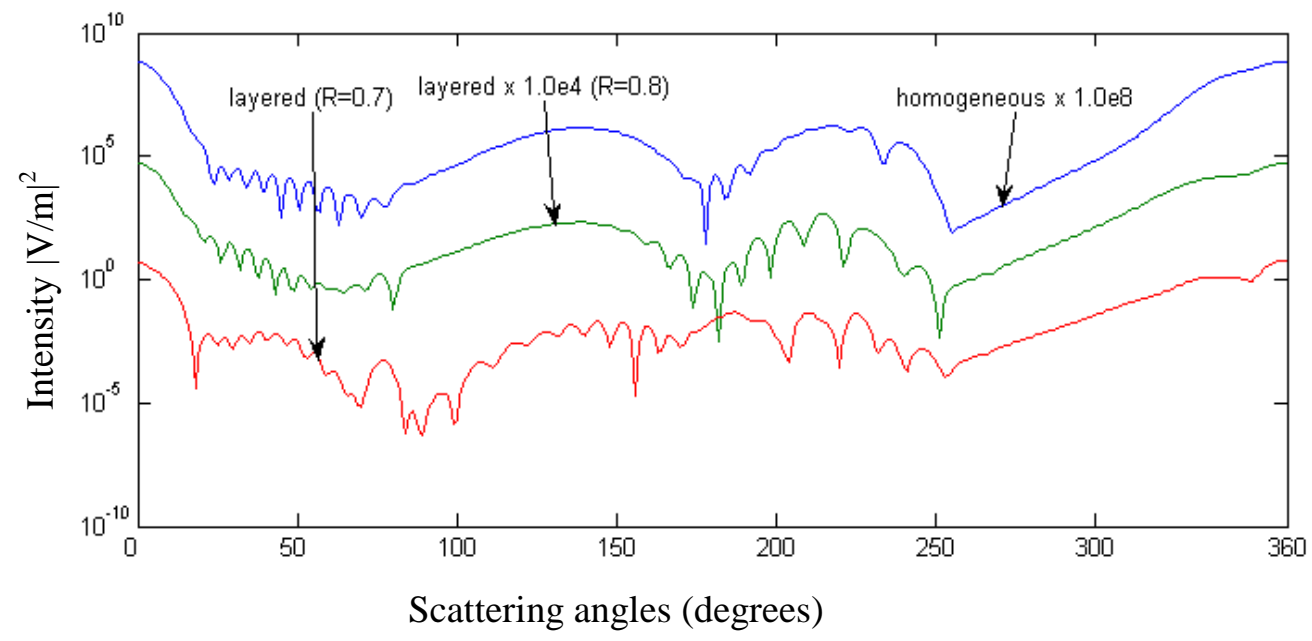

(a)

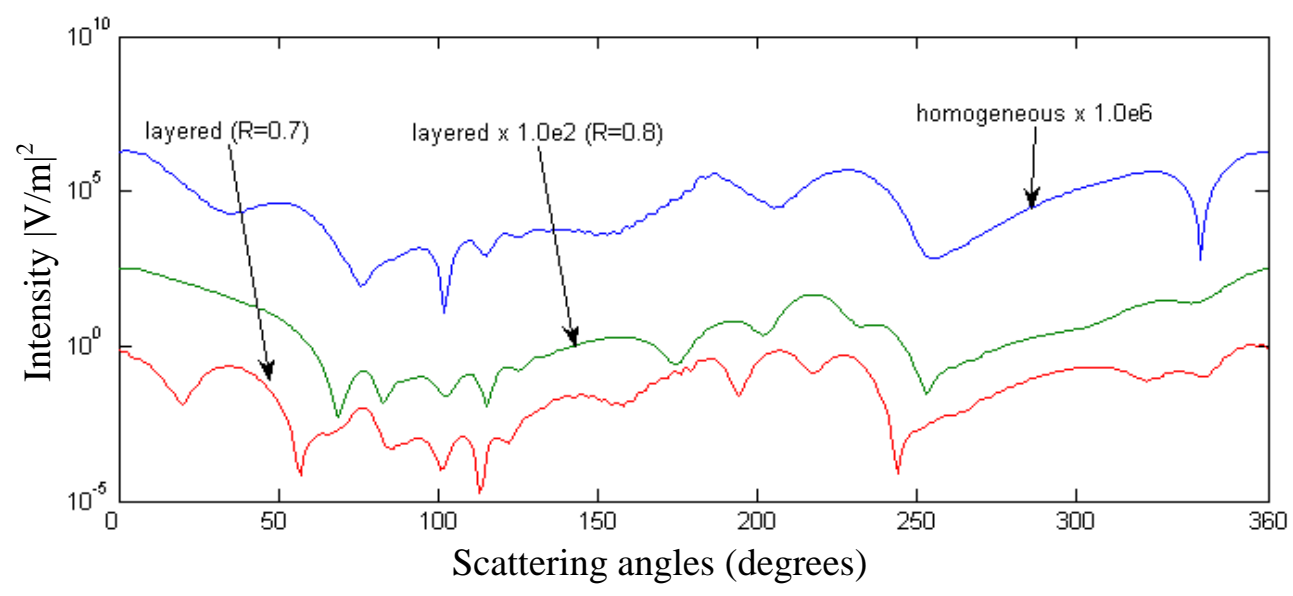

(b)

Fig. 6. Angular scattering intensities for a homogeneous and a coated oblate spheroidal particle illuminated with an off-axis Gaussian beam. All the parameters are the same as in Fig. 5 except that the beam is shifted along the $x$-axis to (a) $x_{o}=0.5 b_{s}$, and (b) $x_{o}=b_{s}$. 


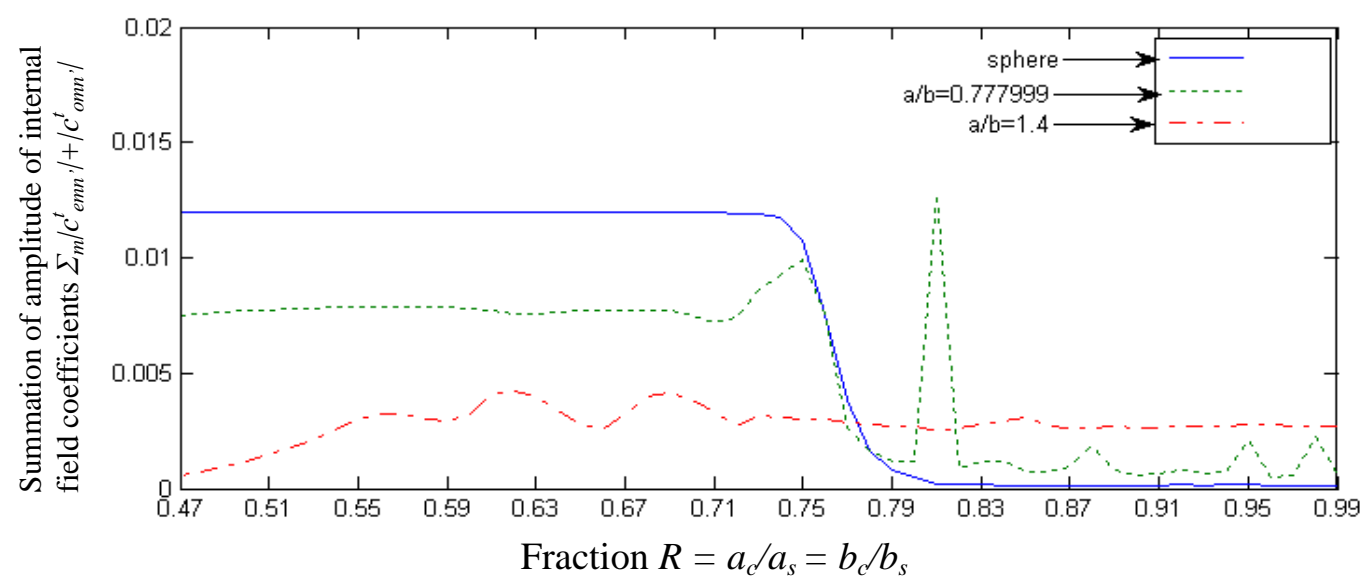

Fig. 7. Summation of the amplitude of the internal field expansion coefficients corresponding to the resonant mode $\mathrm{TE}_{58,1}$ over the azimuthal modes $\Sigma_{\mathrm{m}}\left|\mathrm{c}_{\mathrm{emn}}\right|+\left|\mathrm{c}_{\mathrm{omn}}\right|$ as a function of the fraction $R=a_{d} / a_{s}=b_{d} / b_{s}$ for three different axial ratios; $\rho=1$ (sphere), $\rho=a_{c} / b_{c}=a_{s} / b_{s}=1.4$ (prolate spheroid), and $\rho=a_{c} / b_{c}=a_{s} / b_{s}=0.77799$ (oblate spheroid).

The refractive indices of coated particle are $m_{s}=1.36, m_{c}=1.5$ for the core and shell

respectively. The particle is illuminated with an off-axis Gaussian beam. All parameters of the beam are the same as those in Fig. 3 except that the beam is shifted to $x_{o}=11.098098 \mu \mathrm{m}>>b_{s}, y_{o}=z_{o}=0$.

A Pentium III, $1.7 \mathrm{GHz}$ with $156 \mathrm{MB}$ RAM computer is used for the presented computations. The computation time is relatively small. For example the real computation time for a case from those presented in Fig. 3 is 3 minutes. The off-axis illumination case takes more computation time than that of the on-axis illumination. A one case from those in Fig. 7 takes 13 hours approximately.

\section{CONCLUSIONS}

Angular scattering intensities are calculated for a coated prolate and oblate spheroidal particle illuminated with a Gaussian beam focused at different positions relative to the center of the spheroid. The T-matrix programming codes of a homogeneous spheroid to calculate the elements of the T-matrix and hence the internal and scattered fields are modified to deal with a coated spheroidal particle. Different angular scattering intensities are obtained for different core's radius of three particle's shapes; a sphere, prolate spheroid, and oblate spheroid. The angular scattering intensity is strongly affected by the core's radius in the spheroidal particle case more than that in the case of the sphere either for on- or off-axis illuminations. The computed results may be useful for particle characterization and counting, wafer cleaning, and micro or nanotechnology industries. 


\section{REFERENCES}

[1] Yiping Han, Huayong Zhang, Sun xiangming, "Scattering of shaped beam by an arbitrarily oriented spheroid having layers with nonconfocal boundaries", Applied Physics B Lasers and Optics, vol.84, pp. 485-492, 2006.

[2] E. E. M. Khaled, S. C. Hill, and P. W. Barber, "Light scattering by a coated sphere illuminated with a Gaussian beam", Appl. Opt, Vol. 33, pp. 3308-3314, 1994.

[3] M. I. Mishchenko, L. D. Travis, and A. A. Lacis, Scattering, absorption, and emission of light by small particles, NASA Goddard Institute for Space Studies, New York, 2005.

[4] A. R. Sebak, B. P. Sinha, "Scattering by a conducting spheroidal object with dielectric coating at axial incidence", IEEE Trans. Antennas Propag. Vol. 40, pp. 268-274,1992.

[5] L.-W. Li, M.-S. Leong, T.-S. Yeo, Y.-B. Gan, "Electromagnetic radiation from a prolate spheroidal antenna enclosed in a confocal spheroidal radome", IEEE Trans. Antennas Propag. Vol. 50, pp. 1525-1533, 2002.

[6] J. P. Barton, "Internal and near-surface and scattered electromagnetic fields for a layered spheroid with arbitrary illumination", Appl. Opt. Vol. 40, pp. 3598-3607, 2001.

[7] H. Zhang, and Y. Han," Scattering by a Confocal Multilayered Spheroidal Particle Illuminated by an Axial Gaussian Beam", IEEE, Transactions on Antennas and Propagation, Vol. 53, pp. 1514-1518, 2005.

[8] B. Peterson, and S. Strom, "T-matrix formulation of electromagnetic scattering from multilayered scatterers", Phys Rev D, pp. 2685-2690, 1974.

[9] E. E. M. Khaled, S. C. Hill, and P. W. Barber, "Scattered and internal intensity of a sphere illuminated with a Gaussian beam", IEEE Transaction on Antenna and Propagation, Vol. 41, pp. 295-303, 1993.

[10] A. Quirantes, "A T-matrix method and computer code for randomly oriented, axially symmetric coated scatterers", Journal of Quantitative Spectroscopy \& Radiative Transfer 92, pp. 373-381, 2005.

[11] P. W. Barber, and S. C. Hill, Light Scattering by Particles: Computational Methods, World Scientific, Singapore, 1990.

[12] E. E. M. Khaled, and H. Louka, "Scattered and Internal Intensities of a Homogeneous Dielectric Spheroidal Object Illuminated with an Arbitrary Focused Off-Axis Electromagnetic Beam," accepted for publication in the Journal of Scientific Engineering Sciences (JSES), Assiut University, Assiut, Egypt, July 2009. 


\section{تشتت شعاع جاوسين مركز بواسطة جسيم بيضاوي \\ من مادة عازلة وذو قشرة خارجية \\ قسم الهندسة الكهربائية ـ كلية الهندسة - مالد جامعة أسيوط \\ Esamk54_2000@hotmail.com}

تم في هذا البحث دراسة تشنت شعاع ليزر مركز وعلى شكل جاوسين بواسطة جسيم بيضاوي من مادة

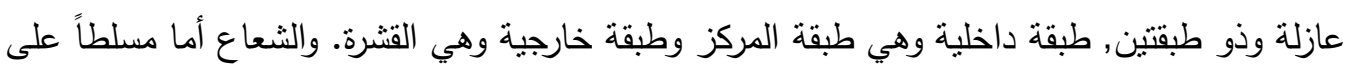

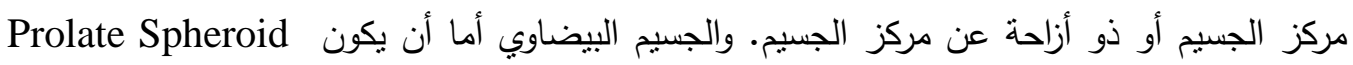

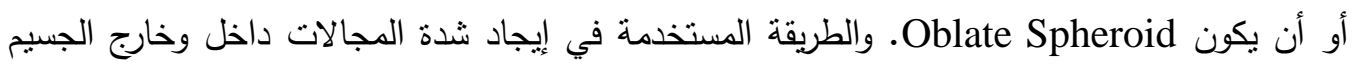
هو أستخدام طريقة التحليل الموجي القاعدي لتحليل الثعاع الساقط الى موجات مستوية قاعدية ثن أستخدام طريقة الـ T-matrix لإيجاد شدة المجالات داخل وخرج الجسيم. ولقد تم تعديل برامج الكمبيوتر التى بنيت من قبل للجسيم المتجانس لأستخدامها في حالة الجسيم ذو الطبقة الخارجية. والنتائج المعطاه هى نتائج محسوبة لجسيم ذو معامل حجمي كبير ولمواضع مختلفة للثنعاع الساقط بالنسبة للجسيم

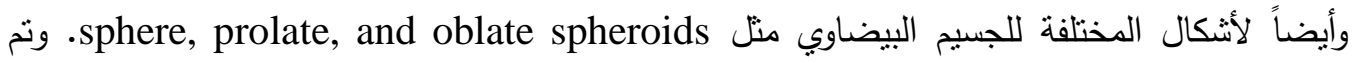
ايضاً اعطاء نتائج لأنصاف أقطار مختلفة لطبقة المركز . وتبين من الدراسة أن التشتت الزاوي بصفة عامة يتأثز بتغير نصف قطر طبقة المركز في حالة الجسيم

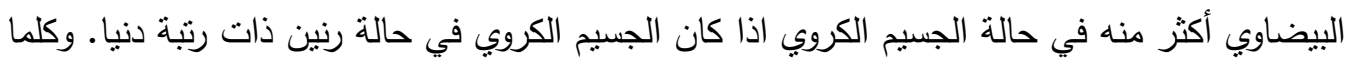

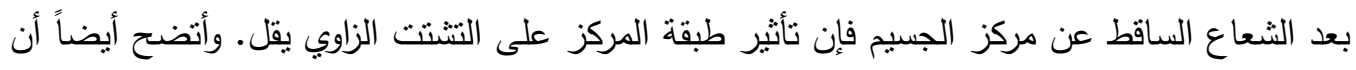

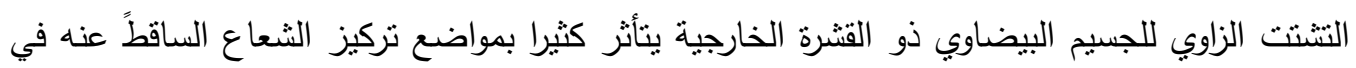

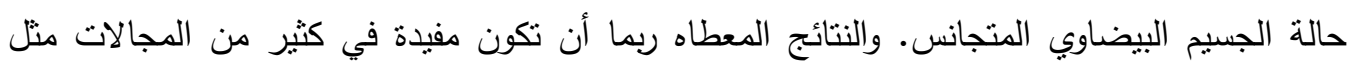

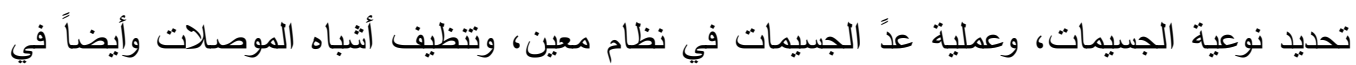
صناعات الميكرو أو النانوتكنولوجي. 